\title{
Elementary radiation patterns in pulsar profiles
}

\author{
Jarosław Dyks and Bronisław Rudak \\ Nicolaus Copernicus Astronomical Center, Toruń, Poland, \\ email: jinx@ncac.torun.pl, bronek@ncac.torun.pl
}

\begin{abstract}
Highly symmetric double features observed in averaged pulsar profiles can be interpreted as the imprint of microscopic radiation beam characteristic of radiative mechanism operating in pulsar magnetosphere. The data put strong constraints on the possible radiation patterns, excluding entire classes of mechanisms, such as those based on parallel acceleration, or those that have complicated beams. Instead, several properties of double features (such as their symmetry, depth, shape, merging rate, large polarisation degree, and the association with bifurcated emission components) are consistent with the extraordinary-mode part of the curvature radiation beam. This shows that double notches are a clear signature of the curvature radiation process. We show that even with the emission process fixed, detailed modelling of double features remains a rather sophisticated and demanding task.
\end{abstract}

Keywords. pulsars: general, pulsars: individual (B1929+10, J0437-4715), radiation mechanisms: nonthermal

\section{Introduction}

Double absorption features (or: double notches) have so far been observed in integrated radio profiles of B1929+10 (Rankin \& Rathnasree 1997), J0437-4715 (Navarro et al. 1997) and B0950+08 (McLaughlin \& Rankin 2004). They are the peculiar 'W'shaped features observed in highly polarised emission. The separation $\Delta$ between the minima of the notches decreases with increasing frequency $\nu$. They have a large depth of $20-50 \%$ (Perry \& Lyne 1985; Rankin \& Rathnasree 1997).

The notches have the symmetry characteristic for relativistic dipole radiation patterns. The basic model of this phenomenon assumes that each point of a laterally extended region locally emits a structured beam of radiation, with a shape determined by the type of radiative process. Hereafter, this angular distribution (directional pattern) of emitted radiation will be called a 'microbeam'. The notches can be observed when our line of sight is passing through a localised, non-emitting part of this region (passage over a spot that is unable to emit detectable radiation).

\section{Double notches as the probe of emission microphysics}

The notches have fairly simple shape: they look like the letter ' $\mathrm{W}$ ', which is mirror symmetric with respect to its centre (see Fig. 2). If the ' $\mathrm{W}$ ' is interpreted as the shape of the microbeam, one has to reject any mechanisms which intrinsically produce microbeams of complicated shape. A neat example of such a model is the 'curvature-drift-driven curvature-radiation maser' in which only selected sub-parts of the usual (non-coherent) curvature radiation beam can be amplified. As shown by Luo \& Melrose (1992), the absorption coefficient can become negative only within those parts of the beam (i.e. for only those directions within the beam), where emissivity decreases for increasing Lorentz factor $\gamma$. Thanks to the curvature drift, which inclines the beam further away from the 


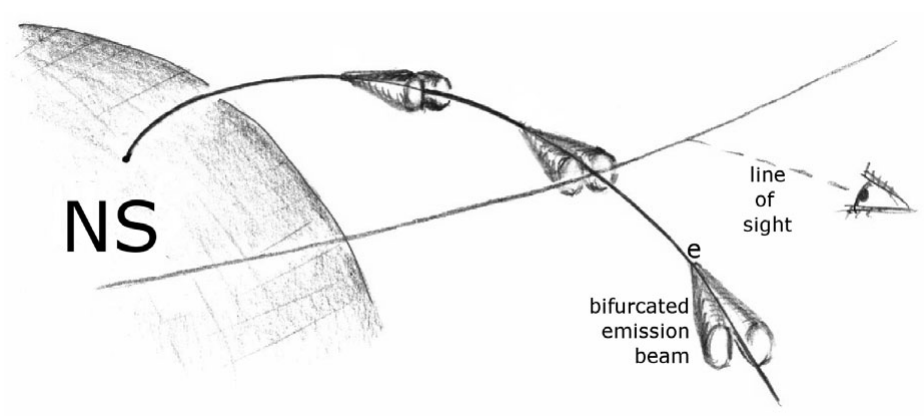

Figure 1. The principle of creating double features in radio pulse profiles: the line of sight traverses through a split-fan emission pattern, produced by outflowing particles that emit a double-lobed beam. Double notches are produced when a stream such as shown above is unable to emit any radiation, but is immersed in a laterally extended emission region.

plane of B-field lines when $\gamma$ increases, negative absorption can occur, but only within two sub-regions of the beam (see Fig. 1b in Luo \& Melrose 1992). These amplified fragments of the beam are localised asymmetrically with respect to the symmetry plane of the noncoherent beam. They are therefore intrinsically asymmetric and cannot be associated with double notches.

The smooth and symmetric shape of double notches then tells us that whatever is the emission mechanism, it does not perform so drastic surgery on the beam's shape, as in the case of the curvature drift maser. Instead, the striking resemblance to the textbook dipole radiation patterns suggests that the coherency mechanism amplifies the beam isotropically, preserving the original, non-coherent shape of the beam.

The angular distribution of dipole radiation from relativistic particles is considered in two limiting cases: when the acceleration vector $\vec{a}$ is parallel to, or perpendicular to, the charge velocity $\vec{v}$. In the case of $\vec{a} \| \vec{v}$ the beam has the obviously structured form of a hollow cone, with $\vec{v}$ along the beam's axis. In the case of $\vec{v} \perp \vec{a}$, the beam can also exhibit a structure that may be attributed to the shape of double notches (see further below).

The parallel acceleration beam $(\vec{v} \| \vec{a})$ is emitted, for example, when longitudinal plasma (Langmuir) oscillations of frequency $\nu_{p}$ are inverse-Compton scattered by electrons with Lorentz factor $\gamma$. This type of a microbeam was initially thought to be associated with the double notches (Dyks, Rudak \& Rankin 2007, hereafter DRR07). Though this idea has finally appeared incorrect, such a model was able to explain the observed merging rate of notches with increasing frequency: $\Delta \sim 1 / \gamma \sim\left(\nu_{p} / \nu\right)^{1 / 2}$. In the case of a wide energy distribution of the scattering electrons, such a model produces intrinsically wide-band emission even from a localised spot in the magnetosphere, which is consistent with the frequency-independent location of double features in pulse profiles (lack of radius-to-frequency mapping, or RFM). Moreover, Lorentz factors of $\gamma \sim 10$, consistent with the measured separation of notches $\Delta \sim$ a few degrees, have been proposed in this model long before the notches have been discovered (Melrose 1978). Nevertheless, such a parallel-ICS model is considered to be not efficient enough to explain the observed large radio fluxes of pulsars, although numerical simulations seem to provide much stronger coherence than is achievable from analytical models (Schopper et al. 2002). When confronted with the observed depth of double notches, the hollow-cone beam of the parallel-acceleration model fails definitely: the beam is unable to produce notches as deep as observed.

As shown in Dyks, Rudak \& Demorest (2010, hereafter DRD10), the depth of modelled notches is very sensitive to the topology of the assumed microbeam. In the case of the 


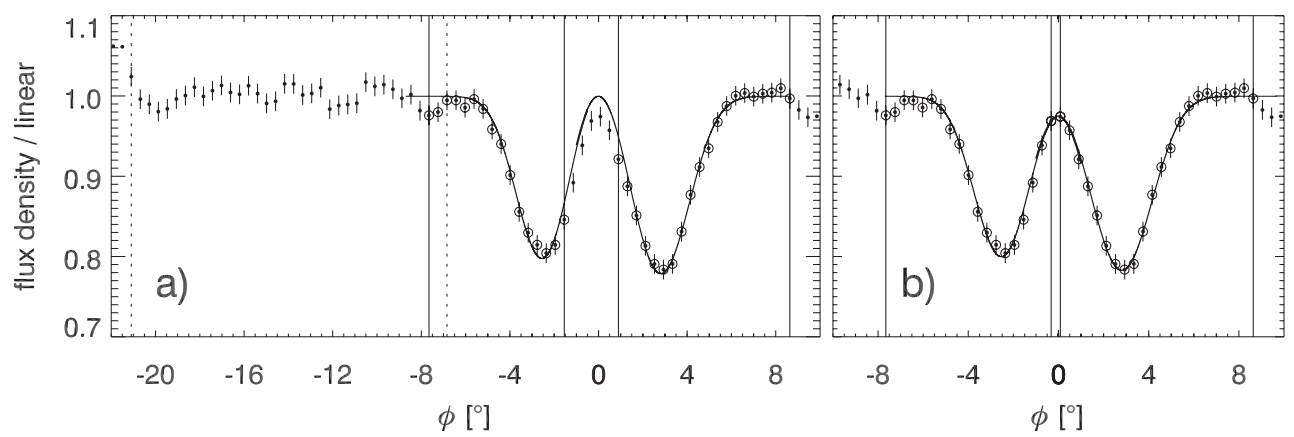

Figure 2. A fit of the curvature radiation beam to double notches in the $327-\mathrm{MHz}$ pulse profile of B1929+10. Each notch is fitted separately, within the left-hand side, and right-hand side pair of solid vertical lines. The pair of dotted verticals marks the phase interval used to remove the linear increase of flux with phase. In panel a) the orthogonal polarisation mode is only used. In panel b) $13 \%$ of parallel mode has been added to match the central data points. The data were collected with the Arecibo Telescope (DRR07); the bandwidth was $25 \mathrm{MHz}$.

hollow-cone beam the minima of the notches are considerably back-lit by the surrounding area of laterally-extended emission region. This makes the notches very shallow, with maximum depth of a few per cent, to be compared with the observed value of more than $20 \%$ for B1929+10. This value has been determined thanks to the interferometric observations of Perry \& Lyne (1985). For J0437-4715, with unknown zero-flux level in its averaged profile, only the upper limit of the depth is known, and estimated to $\sim 50 \%$. This upper limit is close to the actual depth of double notches, if the minimum flux in the averaged profile of this pulsar is close to zero.

It has been shown in DRD10 that the depth approaching $\sim 50 \%$ results from the fact that the emission is intrinsically two-directional, or, that the beam emitted from any local point is double-lobed (Fig. 1). In such a case, at any pulse longitude the observer simultaneously detects radiation from two separate spots in a spatially-extended emission region. At the phase of a minimum in the notches, only one of the two places is nonemitting (or eclipsed), hence the $50 \%$ upper limit for the depth of notches.

The outflowing charges carry the double-lobed beam along the curved magnetic field lines (Fig. 1). This creates a split-fan-shaped beam with strong emissivity at a small angle to the plane of the stream, but with no emission strictly in the plane itself. The split-fan beam model of double notches is then supported by the observation of highly symmetric bifurcated emission components (BECs) in the pulse profiles of J0437-4715 and J1012+5307. In J0437-4715, double notches are actually observed in a trailing wing of a BEC (Navarro et al. 1997).

As shown in Fig. 1, detection of double features in pulsar profiles corresponds to the passage of sightline through the plane of the plasma stream. Therefore, the location of bifurcated features in pulsar profiles is frequency-independent. Double features are then useful to absolutely align profiles observed at different $\nu$ (see figs. 1 and 2 in DRD10). This can be used to precisely determine dispersion measure.

In the case of the curvature radiation $(\vec{a} \| \vec{v})$ the beam can be decomposed into two parts: one which is polarised orthogonally to the plane of electron trajectory and the remaining part, polarised parallel to this plane. In pulsar plasma, only the orthogonallypolarised part (X-mode) can reach the observer, whereas the remaining ordinary-mode part is suppressed and damped (Asseo, Pellat \& Sol 1983; Arons \& Barnard 1986). The extraordinary-mode part of the curvature radiation beam has several properties consistent with the observed properties of double notches: it has the much needed, 


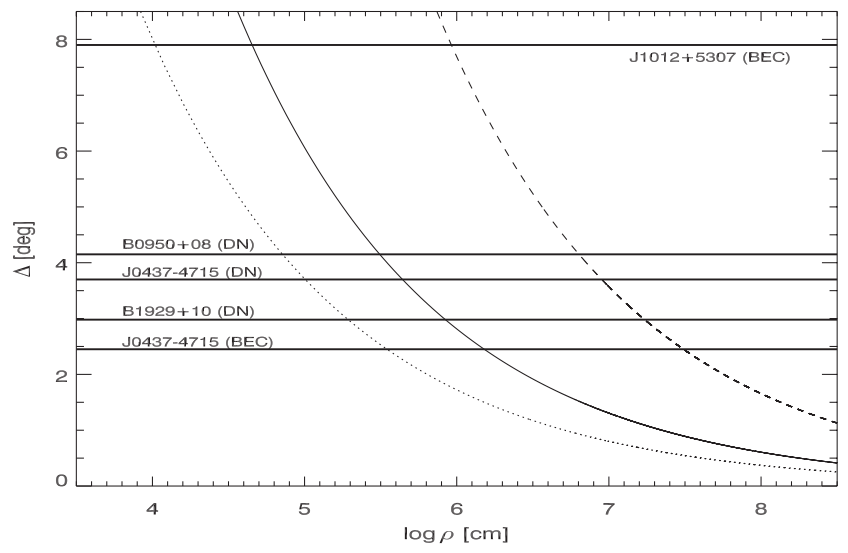

Figure 3. Separation of peaks/dips $\Delta$ at $\nu=1 \mathrm{GHz}$, as a function of curvature radius of electron trajectory $\rho$. The solid curve is for statistically average angles $\zeta=60^{\circ}$ and $\delta_{\text {cut }}=45^{\circ}$. Majority of the values of $\Delta$ measured at $1 \mathrm{GHz}$ (marked with the horizontal lines) are consistent with $\rho \sim 6 \times 10^{5} \mathrm{~cm}$. The dashed curve illustrates the case when one of the angles is small $\left(\zeta=60^{\circ}\right.$, $\left.\delta_{\text {cut }}=15^{\circ}\right)$. The dotted curve is for equatorial viewing and orthogonal cut through the beam $\left(\zeta=90^{\circ}, \delta_{\text {cut }}=90^{\circ}\right.$, i.e. no magnification by the viewing geometry).

double-lobed, and plane-symmetric shape, which allows us to explain the large depth of notches. The beam narrows with increasing frequency at a rate which is consistent with the observations. In the case of the curvature radiation (CR), the angular separation $\Delta$ between the peaks of the beam, varies as $\Delta \propto \nu^{-1 / 3}$, or $\Delta \propto \nu^{-1 / 2}$, depending on whether the low-frequency, or high-frequency part of the spectrum is observed (Jackson 1962). This roughly corresponds to the observed merging rate of double features (see Fig. 7 in Dyks \& Rudak 2012, hereafter DR12).

The extraordinary part of the curvature microbeam successfully reproduces the shape of double notches in the pulse profile of B1929+10. Fig. 2a presents such a fit when no account for the non-zero width of the stream is done. The asymmetry of the notches is attributed to the oblique cut through the stream. When the central maximum of the ' $\mathrm{W}$ ' is lowered down to mimic the finite width of the stream (Fig. 2b) the quality of the fit reaches $\chi^{2} /$ dof $=0.7$ and 0.2 for the left- and right-hand notches, respectively (see DR12 for more details).

The intrinsic size of the $\mathrm{X}$-mode CR beam is $2 \psi=1.72^{\circ}\left(\rho_{6} \nu_{9}\right)^{-1 / 3}$, where $\rho=10^{6} \mathrm{~cm} \times$ $\rho_{6}$ is the curvature radius of electron trajectory, and $\nu_{9}$ is the frequency in GHz. The apparent size of the beam, measurable as the observed separation of peaks/dips, can be approximated with the formula: $\Delta \simeq 2 \psi /\left(\sin \zeta \sin \delta_{\text {cut }}\right)$, where $\zeta$ is the viewing angle between the rotation axis and the line of sight, whereas $\delta_{\text {cut }}$ is the 'cut' angle at which the sightline traverses through the split-fan beam (see Fig. 2 in DR12). The apparent $\Delta$ can then be magnified by the well-known effect of 'not a small angle' $\left(\zeta \ll 90^{\circ}\right)$ as well as because of the oblique passage through the beam $\left(\delta_{\text {cut }} \ll 90^{\circ}\right)$. For statistically-average values of $\zeta=60^{\circ}$ and $\delta_{\text {cut }}=45^{\circ}$, the separations measured at $1 \mathrm{GHz}$ suggest that most of double features corresponds to $\rho_{6}$ between 0.3 and 1 (see Fig. 3 ). This is the curvature of dipolar B-field lines in the closed field line region: for the neutron star radius of $10^{6}$ $\mathrm{cm}$, the smallest $\rho$ available in dipolar magnetosphere of every pulsar is equal to $3.3 \times 10^{5}$ $\mathrm{cm}$, whereas the surface value of $\rho$ in the region emitting at a right angle with respect to the dipole axis is $8.7 \times 10^{5} \mathrm{~cm}$. Some double features are located far from the main pulse of the profile, which is also consistent with emission from the closed field line region. The pulsar J1012+5307 has an exceptionally wide bifurcated emission component (BEC) 


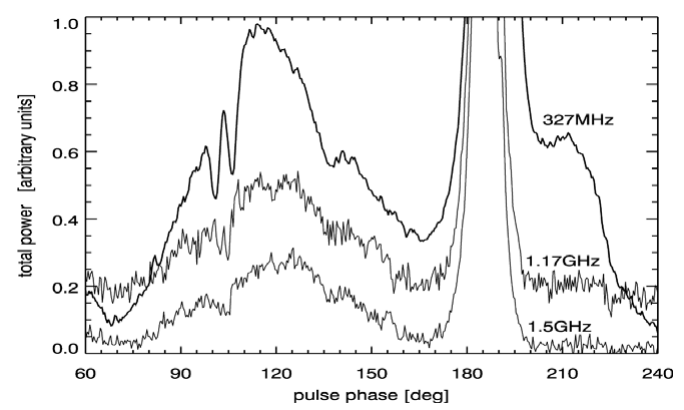

Figure 4. A zoom into averaged pulse profiles of B1929+10 at three different frequencies (all lines present the total flux). Because of narrowing of the non-emitted beam, the clearly double feature at $327 \mathrm{MHz}$ (pulse phase $103.5^{\circ}$ ) evolves into a single feature at $1.5 \mathrm{GHz}$.

which requires that at least one of the viewing parameters is very small (see the dashed line in Fig. 3, calculated for $\left.\zeta=60^{\circ}, \delta_{\text {cut }}=15^{\circ}\right)$.

\section{Complexity of modelling of double features}

The shape of double features can well be reproduced exclusively by the shape of the microbeam, provided the beam is much wider than the non-emitting stream (or the emitting stream, in the case of bifurcated emission components). This is roughly the case shown in Fig. 2. At increasing frequency $\nu$, the microbeam becomes narrower and may become comparable to the stream's width. The resulting shape is then a convolution of the microbeam shape with the profile of plasma density/emissivity across the stream. Numerical simulations show that because of such convolution, the flux at the central maximum in double notches decreases, and the ' $\mathrm{W}$ ' transforms into a single, Ushaped feature. This qualitatively explains the frequency evolution of double notches in B1929+10 (see Fig. 4).

The necessity of the convolution of the beam with the stream profile is just one of several difficulties that need to be taken into account to model double features properly. The second problem is that for an oblique passage through the split-fan beam, double features look generally asymmetric (see fig. 2 in DR12). The degree of this asymmetry is frequency-dependent in the presence of the RFM, i.e. when the minima (or maxima) of emissivity occur at a $\nu$-dependent height. Double features are then symmetric only at a specific 'frequency of symmetry' $\nu_{\mathrm{sym}}$, whereas they look asymmetric at $\nu \neq \nu_{\mathrm{sym}}$. This phenomenon may be responsible for a slight asymmetry of notches shown in Fig. 2 . Such oblique-cut asymmetry also explains the frequency evolution of the pronounced, bifurcated emission component in the profile of J1012+5307 (fig. 5 in Kramer et al. 1999; for more details see DR12). Therefore, to fit double notches precisely, it is required to model full 3D geometry of emission stream, with adjustable parameters describing the RFM law, $r$-dependence of $\rho$, and adjustable cut angle $\delta_{\text {cut }}$.

Some complexity is related directly to the model of the microbeam itself: even for a precisely selected frequency $\nu$ (with infinitely narrow bandwidth) the shape of outer wings of the CR microbeam varies slightly with electron energy $\gamma$. To find a net shape of the beam it is then necessary to integrate over the electron energy distribution. This makes the number of fitting parameters even larger.

Last but not least, the physical model of the curvature microbeam can be improved, by using the curvature radiation pattern for a charge moving in electron-positron plasma. Several properties of this 'charge in plasma' beam are similar to the properties of the 
orthogonally polarised part of the vacuum beam, eg. the double-lobed form with no emission within the plane of trajectory, or $\nu$-dependence of the beam size. However, the shape of this pattern is somewhat different from the orthogonally polarised part of the vacuum beam, which has been used in Fig. 2. This is because the wave induces additional current in the plasma, which modifies the wave equation with respect to the vacuum case. A clear, easy-to-follow derivation of differential equation that describes this beam shape is given in Gil, Lyubarsky \& Melikidze (2004). It will be interesting to use this version of the beam in future modelling efforts.

\section{Conclusions}

Bifurcated emission features observed in averaged radio profiles must be created by the passage of sightline through elongated, split-fan-shaped beams of emission (as opposed to hollow cone beams). Bifurcated 'absorption' features (double notches) are created in a similar way: through observation of an elongated, double 'absorption' structure with geometry similar to the split-fan emission beam.

This interpretation implies that apparently multiconal profiles of some pulsars (eg. the millisecond PSR J0437-4715) actually have spoke-like geometry, with the radiating streams diverging away from the magnetic dipole axis. This explains the lack of RFM in such pulsars, as well as the indentations (dips) at the peaks of such 'not-really-conal' components.

Curvature radiation is a mechanism which intrinsically emits the beam with appropriate properties: double-lobed, narrowing with increasing frequency, and limited to a single polarisation mode, with the latter property consistent with the very large polarisation degree of double features. Therefore, double notches can be considered as a paramount signature of curvature radiation operating in pulsar magnetosphere.

This work has been supported by the National Science Centre grant DEC-2011/02/A/ST9/00256, and by the grant N203 387737 of Polish Ministry of Science and Higher Education. We acknowledge entertaining discussions with Giorgi Melikidze and Janusz Gil.

\section{References}

Arons, J. \& Barnard, J. J. 1986, ApJ, 302, 120

Asseo, E., Pellat, R., \& Sol, H. 1983, ApJ, 266, 201

Dyks, J. \& Rudak, B. 2012, MNRAS, 420, 3403 (DR12)

Dyks, J., Rudak, B., \& Demorest, P. 2010, MNRAS, 401, 1781 (DRD10)

Dyks, J., Rudak, B., \& Rankin, J. M. 2007, A $\& A, 465,981$ (DRR07)

Gil, J., Lyubarsky, Y., \& Melikidze, G. 2004, ApJ, 600, 872

Jackson, J. D. 1962, Classical electrodynamics, John Wiley \& Sons, Inc., New York

Kramer, M., Lange, C., Lorimer, D., Backer, D. C., Xilouris, K. M., Jessner, A., \& Wielebinski, R. 1999, ApJ, 526, 957

Luo, Q. \& Melrose, D. B., 1992, MNRAS, 258, 616

McLaughlin, M. A. \& Rankin, J. M. 2004, MNRAS, 351, 808

Melrose, D. B. 1978, ApJ, 225, 557

Navarro, J., Manchester, R. N., Sandhu, J. S., Kulkarni, S. R., \& Bailes, M. 1997, ApJ, 486, 1019

Perry, T. E. \& Lyne, A. G. 1985, MNRAS, 212, 489 (PL85)

Rankin, J. M. \& Rathnasree, N. 1997, J. Astrophys. Astron., 18, 91

Schopper, R., Ruhl, H., Kunzl, T. A., \& Lesch, H. 2002, MPE Report 278, 193 